\title{
An uncommon presentation of osteosarcoma
}

\author{
Mantha Satya Padmaja', Sourin Bhuniya², Suprava Naik ${ }^{3}$, Mukund Sable ${ }^{4}$, Sudip Ghosh², \\ Prasanta Raghab Mohapatra ${ }^{2}$
}

${ }^{1}$ Pulmonary Medicine and Critical Care, All India Institute of Medical sciences, Bhubaneswar, India

${ }^{2}$ Department of Pulmonary Medicine and Critical Care, All India Institute of Medical Sciences, Bhubaneswar, India

${ }^{3}$ Department of Radiodiagnosis, All India Institute of Medical sciences, Bhubaneswar, India

${ }^{4}$ Department of Pathology, All India Institute of Medical Sciences, Bhubaneswar, India

A 20-year old female presented to our department with complaints of sudden-onset dyspnea and right-sided chest pain for the last month. The patient was started on empirical anti-tuberculosis therapy by a local physician, but there was no respite from the symptoms and she deteriorated clinically. A detailed patient history revealed that she had pain and swelling over the right knee, left arm, as well as vague complaints of generalized body aches for the last 5 months. On clinical examination, she had tachycardia and tachypnoea with an oxygen saturation of $90 \%$ at room air. There were enlarged, hard, and non-tender lymph nodes over the right axilla $(2 \times 2 \mathrm{~cm})$ and bilateral inguinal regions $(2 \times 1 \mathrm{~cm})$. Lung auscultation revealed dull notes and decreased breath sounds over the left hemithorax. Examination of the lower limbs revealed a diffuse, tender swelling over the right distal thigh. Pleural fluid analysis was exudative with ab ADA of 24 IU/L, and without evidence of any abnormal cells. Radiographic imaging of the right knee was suggestive of an osteogenic malignancy (Figure 1). Serum alkaline phosphatase level was also elevated. Contrast-enhanced computed tomography (CECT) of the thorax and abdomen (Figure 1) revealed extensive calcification of bilateral pleurae, mediastinum, hila and pulmonary vessels. There were multiple enlarged and calcified mediastinal and abdominal lymph nodes along with a calcified left adrenal mass. Excision biopsy of the right axillary lymph node showed sheets of malignant cells surrounding the malignant osteoid with brisk mitotic activity. Immunohistochemical studies showed that the tumour cells were diffusely and strongly immunopositive for Vimentin, while immunonegative for CK, LCA, SALL4, Desmin, Myogenin, S100 and HMB45 (Figure 2). Thus, a diagnosis of metastatic osteosarcoma was made. The patient was critically ill, rapidly progressed to respiratory failure and finally died in the intensive care unit.

The most common primary bone malignancy is osteosarcoma, with the peak incidence in the second decade of life. Around $10 \%$ of patients present with distant metastases. The most common sites of metastasis are the lungs and bone, while the unusual sites of metastasis include the brain, abdominal viscera, lymph nodes, pleura, pericardium and the oral cavity [1]. Analysis of an autopsy database in Japan showed 12.1\% involvement of pleura in patients who died of metastatic osteosarcoma. However, extensive involvement of pleura in primary osteosarcoma during life is very rare. Extensive involvement of pleura may occur either from hematogenous or direct spread from the underlying lung. In our case, bilateral extensive calcification of the pleura was probably due to hematogenous spread from the primary lesion in the right leg.

Regional lymph node involvement in osteosarcoma is rare with reported incidence rates ranging from $<1 \%$ to $10 \%$. The incidence of lymph node metastasis as reported by the Co-operative Osteosarcoma Study Group (COSS) is $0.8 \%$, whereas other series reported clinically detectable lymph node metastasis to be less than $4 \%$ at initial presentation [2,3]. Patients diagnosed with osteosarcoma who have regional lymph node metastasis have a poor overall survival when compared to those without regional node involvement [4]. In one series, the median survival rate reported in this group was 8.5 months after diagnosis, which was similar to those with distant metastasis. Our patient had predominantly metastasis to the pleura and lymph nodes, which is usually rare. Regional lymph node involvement can serve as an adverse prognostic indicator independent of other known prognostic factors in osteosarcoma.

A high index of suspicion and meticulous clinical examination is essential for early diagnosis in cases of undiagnosed pleural effusion and peripheral lymphadenopathy.

Address for correspondence: Sourin Bhuniya, Department of Pulmonary Medicine And Critical Care, All India Institute of Medical Sciences, Bhubaneswar, India; e-mail: sbhuniya@hotmail.com

DOI: 10.5603/ARM.2020.0121

Received: 26.01.2020

Copyright (C) 2020 PTChP

ISSN 2451-4934

Conflict of interest: None declared 

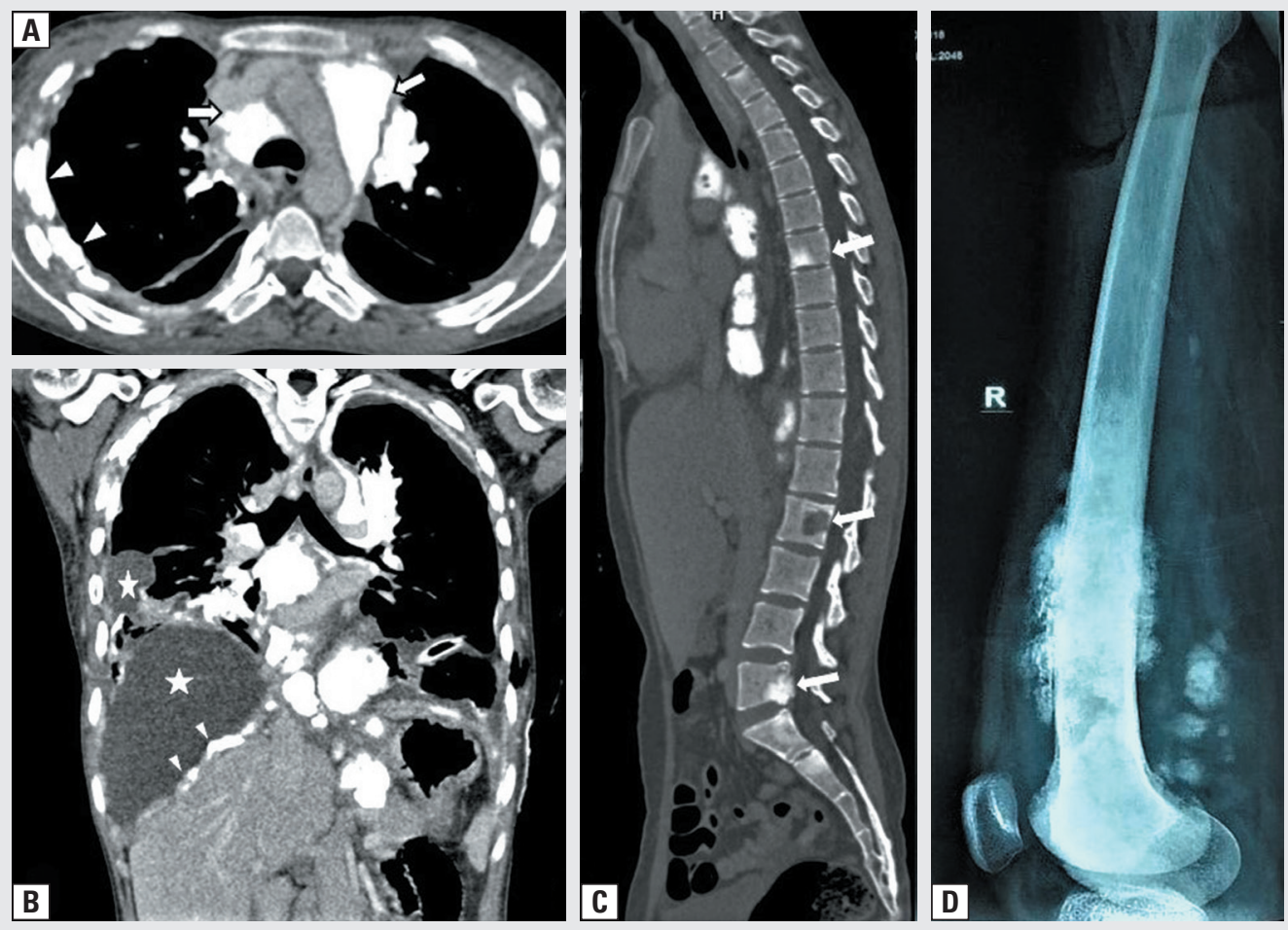

Figure 1. Axial CECT at the level of aortic arch (A.) shows multiple large calcified paraaortic and pre-tracheal lymph nodes (arrows), irregular right sided pleural thickening and calcification (arrowheads). Coronal (B.) and sagittal bone window (C.) reformatted images show extensive mediastinal, perivascular, gastrohepatic metastatic calcified lymphadenopathy, extensive pleural calcification including bilateral diaphragmatic pleura (narrow arrowheads in B.), multiple bilateral loculated pleural effusion (stars in B.), lytic and sclerotic metastasis in vertebrae (arrows in C.). In the same patient, $x$-ray of right thigh lateral view (D.) shows bone tumour with aggressive periosteal reaction at distal femoral metaphysis
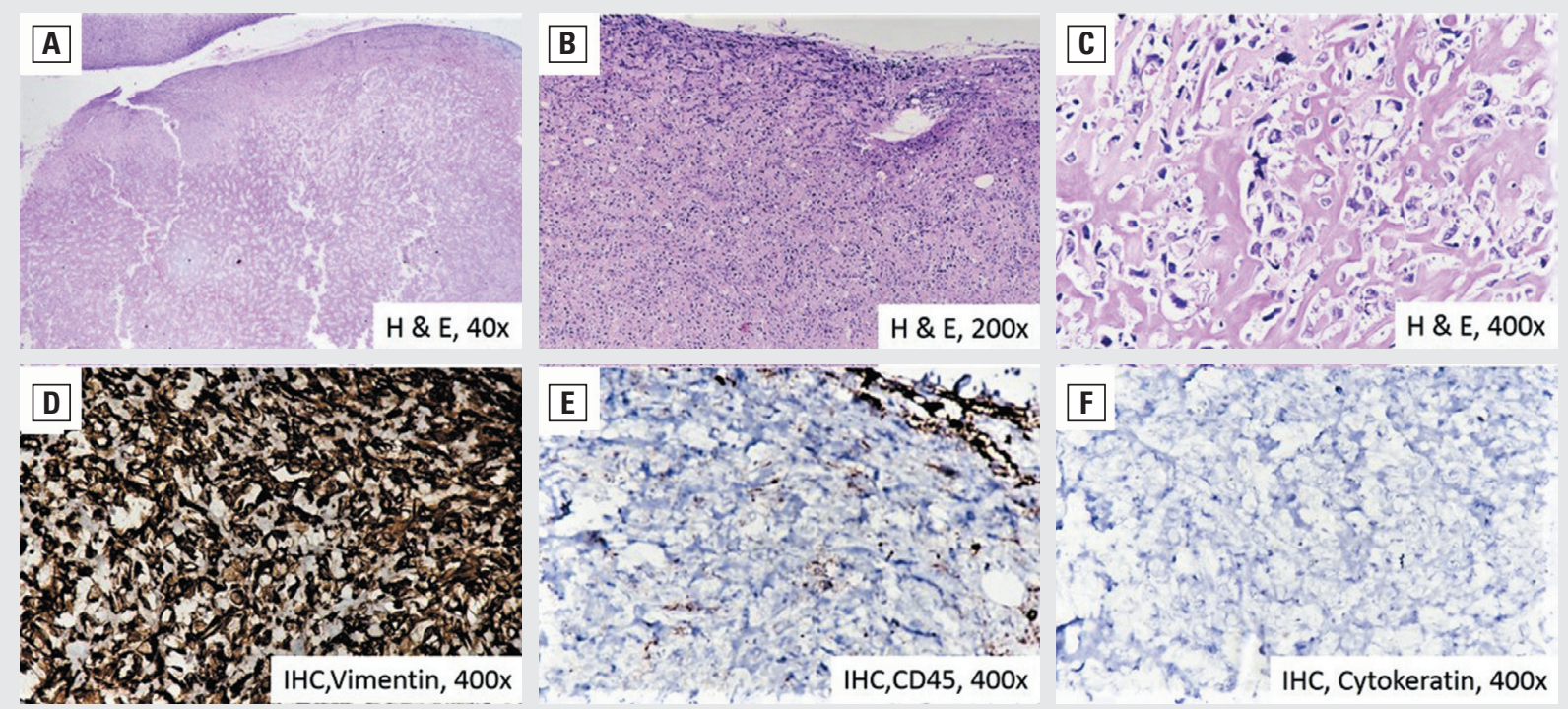

Figure 2. Histopathological examination of lymph node shows complete replacement of lymph node by a tumour tissue, with peripheral rim of lymphoid cells (A., B.). The tumour shows atypical cells with extensive osteoid formation (C.). The tumour was immunopositive for vimentin (D.), while negative for CD45 (E.) and cytokeratin (F.) 


\section{References:}

1. Nouyrigat P, Berdah JF, Roullet B, et al. Osteosarcoma with calcified regional lymph nodes. Pediatr Radiol. 1993; 23(1): 74-75, doi: 10.1007/BF02020235, indexed in Pubmed: 8469605.

2. Bielack SS, Kempf-Bielack B, Delling G, et al. Prognostic factors in high-grade osteosarcoma of the extremities or trunk: an analysis of 1,702 patients treated on neoadjuvant cooperative osteosarcoma study group protocols. J Clin Oncol. 2002; 20(3): 776-790, doi: 10.1200/JCO.2002.20.3.776, indexed in Pubmed: 11821461.

3. Meyers PA, Heller G, Healey JH, et al. Osteogenic sarcoma with clinically detectable metastasis at initial presentation. J Clin Oncol. 1993; 11(3): 449-453, doi: $10.1200 / \mathrm{JCO} .1993 .11 .3 .449$, indexed in Pubmed: $\underline{8445419}$.

4. Thampi S, Matthay KK, Goldsby R, et al. Adverse impact of regional lymph node involvement in osteosarcoma. Eur J Cancer. 2013; 49(16): 3471-3476, doi: 10.1016/j.ejca.2013.06.023, indexed in Pubmed: 23867123. 